\title{
Age not an issue for HoLEP
}

Clinicians from the Mayo Clinic, Phoenix, AZ, USA, have shown that holmium laser enucleation of the prostate (HoLEP) is a safe and effective therapy for $\mathrm{BPH}$, regardless of age. In a retrospective review of all patients with lower urinary tract symptoms (LUTS) resulting from prostate enlargement who were treated with HoLEP at their clinic over a 4-year period, Chinedu and colleagues found that overall morbidity, hospital stay, and 1-year functional outcomes were similar for patients aged 50-59 years $(n=22), 60-69$ years $(n=91), 70-79$ years $(n=153)$, and $\geq 80$ years $(n=45)$.

Although anticoagulation medication use and mean enucleated prostate weight were significantly greater in the older cohorts, there were no differences between the groups in terms of mean haemoglobin change, length of hospital stay (1.181.68 days), or PSA reduction at 6 months. Patients aged $\geq 80$ years were more likely to need a blood transfusion after surgery and morcellation time was slightly longer in the older groups, but these effects did not reach statistical significance.

Encouragingly, patients from all groups showed improvement in their LUTS at first follow-up visit and both postvoid residual urine volume and International Prostate Symptom Score (IPSS) were similar for all age groups at 6 weeks. All groups demonstrated an improvement in their IPSS and Qmax throughout the duration of their follow-up, although patients in the oldest group had a consistently lower Qmax than those in the three younger groups (potentially owing to a higher prevalence of preoperative urinary retention). Importantly, rates of complete continence ( 0 pads) at 1 year were similar for all age groups (100\%, $95 \%, 93 \%$, and $88 \%$ for patients aged 50-59 years, 60-69 years, 70-79 years, and $\geq 80$ years, respectively).

"For the first time, we see a treatment modality that works well for patients from all age groups, independent of prostate size," says Mitchell Humphreys, lead investigator of the trial. "With traditional $\mathrm{BPH}$ treatment options, prostate size is a limiting factor and numerous studies have indicated that age is an independent predictor of poor outcome. For HoLEP, on the other hand, we have shown that outcomes are independent of age. Moreover, the older patients in our study were sicker (with more comorbidities), on significantly more anticoagulation

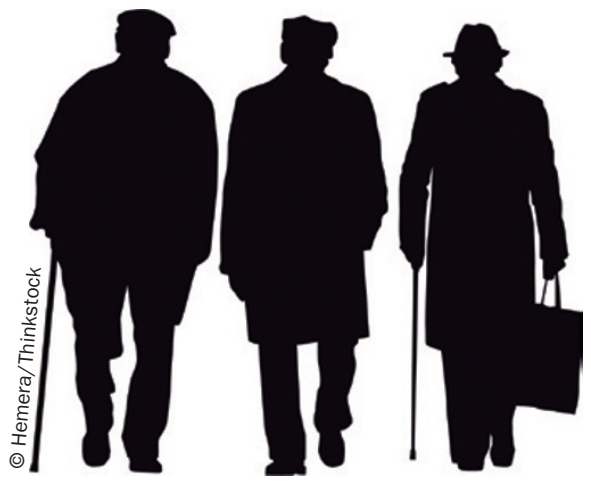

medication at the time of surgery (with no significantly increased risk of transfusion), had bigger prostates, and reported more baseline incontinence."

A common concern with HoLEP is the difficulty of the procedure and the learning curve of the surgeon. However, Chinedu et al. evaluated data from all consecutive patients, including those treated during the learning curve. "Patients have not been excluded to make the data 'look better," says Humphreys. "Our study is a true reflection of the expected HoLEP experience."

Melanie Clyne

Original article Chinedu, 0. et al. Age-stratified outcomes of holmium laser enucleation of the prostate. BJU Int. doi:10.1111/bju.12063 